\title{
Considerations arising from requests from patients for a bilateral mastectomy who are eligible for breast-conserving surgery: Factors weighing for and against performing the operation
}

\author{
ALESSANDRO FANCELLU \\ Department of Clinical and Experimental Medicine, Unit of General Surgery 2-Clinica Chirurgica, \\ University of Sassari, I-07100 Sassari, Italy
}

Received December 9, 2015; Accepted May 18, 2016

DOI: $10.3892 / \mathrm{ol} .2016 .4641$

\begin{abstract}
Rates of mastectomy and contralateral prophylactic mastectomy (CPM) are increasing in patients with unilateral breast cancer who would be candidates for receiving breast-conserving surgery, according to recent studies. One reason for this finding is the entitlement of patients to choose to undergo a bilateral mastectomy as a surgery option. The present case report details the case of a 52-year-old woman, eligible for breast conservation, who had no high risk of developing a contralateral breast cancer, but who asked for a bilateral mastectomy and immediate reconstruction instead of breast-conserving surgery. The request for a bilateral mastectomy was declined on the basis that it would be unnecessary for the patient, and an excessive intervention; the patient subsequently obtained an independent second opinion, and she underwent a bilateral mastectomy with implant reconstruction prior to receiving wound care and follow-up at our institution. At follow-up 1 year later, the patient complained of sensations of general discomfort, sexual difficulties and a loss of sensation in the nipples. On the basis of the clinical outcomes of the bilateral mastectomy and the patient's experience of the operation, the present case study highlights a need for surgeons to clearly inform patients presenting with unilateral breast cancer about the attendant risks and real benefits of bilateral mastectomy, in order to reach a mutual decision in terms of health care that is not fuelled by anxiety or an overestimation of the risk of developing secondary cancer. Bilateral mastectomy should be attentively evaluated as an option only in patients with a higher-than-average risk of developing contralateral breast cancer.
\end{abstract}

Correspondence to: Professor Alessandro Fancellu, Department of Clinical and Experimental Medicine Unit of General Surgery 2-Clinica Chirurgica, University of Sassari, Viale Le San Pietro 43, I-07100 Sassari, Italy

E-mail: afancel@uniss.it

Key words: prophylactic bilateral mastectomy, patients' choice, breast conserving surgery

\section{Introduction}

Local treatment of early breast cancer has changed during the last decade. Although the detection of small non-palpable tumors is becoming more and more frequent, recent literature reports that rates of unilateral and contralateral prophylactic mastectomy (CPM) are increasing in patients who are candidates to breast conserving surgery (1-4). The present case study details a recently observed case at our institution, with the aim of focusing on several important issues in the setting of CPM in patients with unilateral breast cancer.

\section{Case report}

A 52-year-old woman was referred to our institution following a diagnosis of screen-detected breast cancer. Mammography and ultrasonography demonstrated a $0.8 \mathrm{~cm}$ spiculated mass in the lower outer quadrant of her left breast (Figs. 1 and 2). A core-biopsy showed an infiltrating ductal carcinoma of grade 2 . The tumor was non-palpable on physical examination, and there were neither palpable masses of the contralateral breast nor axillary lymph node enlargement.

The patient was a mother of two sons who underwent natural menopause at the age of 49. She had an unremarkable medical history. The patient did not have any prior family history of breast or ovarian cancer and was being medically treated for hypertension. In response to the diagnosis of breast cancer, the patient requested a bilateral mastectomy. After having visited the patient and assessing her medical records, breast-conservation surgery plus sentinel node biopsy followed by radiation therapy was recommended for the patient, clarifying for her that, in the considered opinion of the surgeon, the patient had a very low probability of subsequently developing a contralateral breast cancer.

The patient maintained that she considered bilateral mastectomy and immediate reconstruction would be her preferred option. In response to her appeal, after multidisciplinary team discussions, breast-conserving surgery was again proposed as the course of treatment; bilateral mastectomy was not considered to be viable as an option.

In response to the recommendation from our medical team, the patient elected to ask for a second, independent opinion 

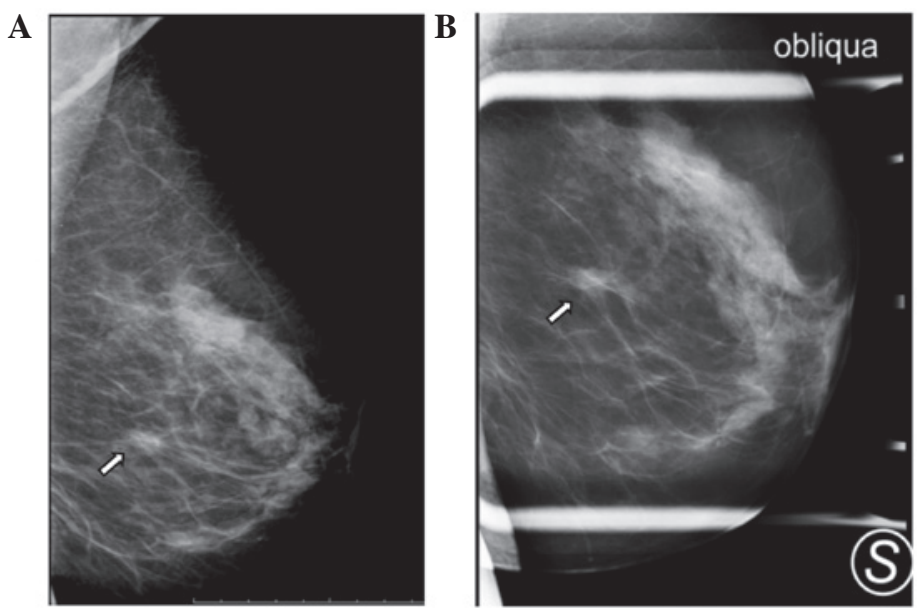

Figure 1. Mammograms showing an irregular, spiculated mass in the lower outer quadrant of the left breast (arrow). (A) Lateral projection; (B) oblique projection.
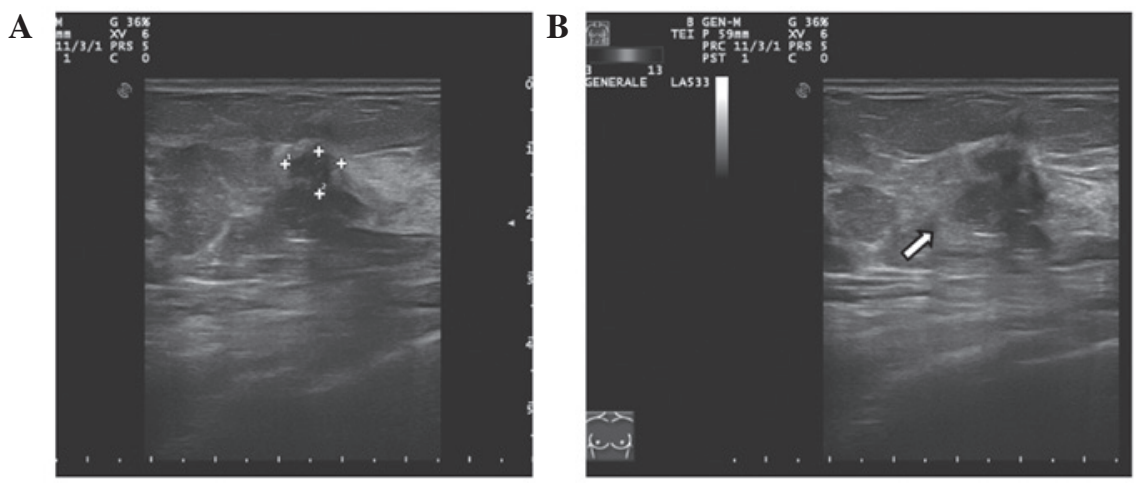

Figure 2. (A and B) Ultrasonography, showing two different views of an hypoechoic mass with irregular margins, measuring $0.8 \times 0.7 \mathrm{~mm}$.

from another surgeon working at a different institution. The patient then underwent a bilateral nipple-sparing mastectomy, with sentinel node biopsy and immediate implant reconstruction. An histological examination revealed no additional foci of cancer in the ipsi- or contralateral breast, other than the index tumor. The sentinel node biopsy was negative.

Subsequently, the patient returned to our institution for wound care and postoperative oncological consultation. The patient commenced endocrine therapy, and was initially satisfied with the cosmetic results of the bilateral reconstruction. At six months following the surgery, the patient returned for a follow-up visit, complaining of occasional bilateral breast pain and an almost complete loss of sensation in her nipples. At the one-year follow-up visit, the patient disclosed a general sense of discomfort linked to the surgical operation, and complained of difficulties with her sexual life. The patient expressed an opinion that she had begun to doubt the necessity of having undergone a bilateral mastectomy, and considered that breast-conserving surgery may been preferable as the treatment option.

\section{Discussion}

For breast surgeons and their patients, the decision-making process has become more and more complex in the last decade. Rates of mastectomy are growing in patients with breast cancer who are eligible for breast conservation (2-5). In addition, rates of CPM are also on the rise for both invasive and in situ cancer $(1,6,7)$. There are several reasons for these phenomena, including the increasing use of preoperative MRI, the growing employment of immediate post-mastectomy reconstruction, improvements in cosmetic results with the relatively new techniques of skin- and nipple-sparing mastectomy, the increasing use of genetic testing, and last but not least, the patients' entitlement to choose their preferred surgery option (6-12).

In the present case study, the recommended surgical option was to perform breast-conserving surgery followed by radiation therapy. A bilateral mastectomy was considered to be an unnecessary and excessive intervention. In fact, it should be recognized that the annual lifetime risk of contralateral breast cancer is $\sim 0.5-1 \%$, and the cumulative 10 year risk is $\sim 4-5 \%(2,5)$. Furthermore, the risk of developing a second, fatal breast cancer is even lower. In a recent large series study, occult breast cancer was identified in $6 \%$ of patients undergoing CPM (12). In particular, there are specific situations in which the risk of developing a contralateral cancer is higher than that reported above, such as familiarity for breast cancer, axillary lymph node involvement, being under 50 years of age at the time of diagnosis, previous chest wall irradiation, and the presence of genetic mutations linked with hereditary breast cancer (1,3-5). Furthermore, it is known that patients 
with BRCA1/2 mutations have a substantially higher risk of contralateral cancer compared with sporadic counterparts, accounting for $12-20 \%$ within 5 years of initial diagnosis $(1,13)$.

In the present case study, the patient had none of those risk factors. Our multidisciplinary breast cancer team considered that they had explained exhaustively to the patient the benefits and risks of bilateral mastectomy, focusing on the issue that the risk of a second breast neoplasm was very low. It is paradoxical that an increasing number of women are choosing to undergo bilateral mastectomy in a setting in which rates of contralateral breast cancer are decreasing, largely as a consequence of adjuvant endocrine therapy $(5,14)$. Benefits of contralateral mastectomy include the diminishing need for clinical and imaging surveillance, cosmetic symmetry in selected cases, and a decreased risk of developing contralateral breast cancer (1). However, the latter should not be considered as an outcome from an oncological point of view. In fact, novel techniques or approaches in surgical oncology should be directed towards survival benefits rather than a simple risk reduction. It is important to highlight that no studies in the literature have demonstrated an advantage in terms of survival by performing a contralateral mastectomy in patients with unilateral breast cancer, except (probably) in the scenario of germline mutations in breast-cancer susceptibility genes $(1,2,6,15)$.

In conclusion, in the setting of unilateral breast cancer eligible for breast conservation, bilateral mastectomy should be attentively evaluated as an option only in patients with a higher-than-average risk of developing a contralateral breast cancer.

\section{References}

1. Tracy MS, Rosenberg SM, Dominici L and Partridge AH: Contralateral prophylactic mastectomy in women with breast cancer: Trends, predictors, and areas for future research. Breast Cancer Res Treat 140: 447-452, 2013.

2. Stucky CC, Gray RJ, Wasif N, Dueck AC and Pockaj BA: Increase in contralateral prophylactic mastectomy: Echoes of a bygone era? Surgical trends for unilateral breast cancer. Ann Surg Oncol 17 (Suppl 3): S330-S337, 2010.
3. Kummerow KL, Du L, Penson DF, Shyr Y and Hooks MA: Nationwide trends in mastectomy for early-stage breast cancer. JAMA Surg 150: 9-16, 2015.

4. Adkisson CD, Bagaria SP, Parker AS, Bray JM, Gibson T, Thomas CS, Heckman MG and McLaughlin SA: Which eligible breast conservation patients choose mastectomy in the setting of newly diagnosed breast cancer? Ann Surg Oncol 19: 1129-1136, 2012.

5. Portschy PR and Tuttle TM: Rise of mastectomy. J Surg Oncol 107: 563-564, 2013

6. Soran A, Kamali Polat A, Johnson R and McGuire KP: Increasing trend of contralateral prophylactic mastectomy: What are the factors behind this phenomenon? Surgeon 12: 316-322, 2014.

7. Janssen S, Holz-Sapra E, Rades D, Moser A and Studer G Nipple-sparing mastectomy in breast cancer patients: The role of adjuvant radiotherapy (Review). Oncol Lett 9: 2435-2441, 2015.

8. Fancellu A, Soro D, Castiglia P, Marras V, Melis M, Cottu P, Cherchi A, Spanu A, Mulas S, Pusceddu C, et al: Usefulness of magnetic resonance in patients with invasive cancer eligible for breast conservation: A comparative study. Clin Breast Cancer 14: 114-121, 2014.

9. Fancellu A, Turner RM, Dixon JM, Pinna A, Cottu P and Houssami N: Meta-analysis of the effect of preoperative breast MRI on the surgical management of ductal carcinoma in situ. Br J Surg 102: 883-893, 2015.

10. Agarwal S, Agarwal S, Neumayer L and Agarwal JP: Therapeutic nipple-sparing mastectomy: Trends based on a national cancer database. Am J Surg 208: 93-98, 2014.

11. King TA, Sakr R, Patil S, Gurevich I, Stempel M, Sampson M and Morrow M: Clinical management factors contribute to the decision for contralateral prophylactic mastectomy. J Clin Oncol 29: 2158-2164, 2011.

12. King TA, Gurevich I, Sakr R, Patil S, Stempel M and Morrow M: Occult malignancy in patients undergoing contralateral prophylactic mastectomy. Ann Surg 254: 2-7, 2011.

13. Verhoog LC, Brekelmans CT, Seynaeve C, van den Bosch LM, Dahmen G, van Geel AN, Tilanus-Linthorst MM, Bartels CC, Wagner A, van den Ouweland A, et al: Survival and tumour characteristics of breast cancer patients with germline mutations of BRCA1. Lancet 351: 316-321, 1998.

14. Rosenberg SM, Sepucha K, Ruddy KJ, Tamimi RM, Gelber S, Meyer ME, Schapira L, Come SE, Borges VF, Golshan M, et al: Local therapy decision-making and contralateral prophylactic mastectomy in young women with early-stage breast cancer. Ann Surg Oncol 22: 3809-3815, 2015.

15. Chung A, Huynh K, Lawrence C, Sim MS and Giuliano A: Comparison of patient characteristics and outcomes of contralateral prophylactic mastectomy and unilateral total mastectomy in breast cancer patients. Ann Surg Oncol 19: 2600-2606, 2012. 